\title{
Time-Reversal Violation in the Nucleon and the Nucleus
}

\section{Ubirajara van Kolck*}

Institut de Physique Nucléaire, Université Paris-Sud, CNRS/IN2P3, 91406 Orsay Cedex, France

E-mail: vankolck@ipno.in2p3.edv

and

Department of Physics, University of Arizona, Tucson, AZ 85721, USA

E-mail: vankolck@physics.arizona.edu

I describe the formalism for the calculation of nucleon and nuclear parity- and time-reversalviolating form factors stemming from operators of dimension up to six in the Standard Model Lagrangian. I argue that proposed measurements of light nuclear electric dipole moments would give valuable information about the source of time-reversal violation.

Sixth International Conference on Quarks and Nuclear Physics

April 16-20, 2012

Ecole Polytechnique, Palaiseau, Paris

\footnotetext{
* Speaker.
} 


\section{Introduction}

It was perhaps appropriate that one of the very last talks in this intense and diverse conference in the city of lights dealt with time-reversal violation $(T)$ and, in particular, the way strongly interacting particles react to electromagnetic fields. My talk had nothing directly to do with the arrow of time; instead I looked to the future in the form of the exciting experimental program - partly in progress, partly in planning - to probe the electric dipole moments (EDMs) of the nucleon and of light nuclei. My goal was to convey why I think this program is very promising.

Parity $(P)$ is the unitary transformation that reverses the sign of all space coordinates while keeping time unchanged. Analogously, time reversal $(T)$ reverses the sign of time keeping space unchanged, but being anti-unitary it also complex-conjugates all numbers. $T$ per se is of course not new. (For a more complete set of references than I have room to provide, I refer you to the many excellent reviews on the subject, for example Ref. [1].) Its manifestation in flavor-changing processes involving mesons with strangeness, charm and bottomness is relatively well understood as a result of a phase in the Cabibbo-Kobayashi-Maskawa (CKM) matrix arising from the existence of at least three families of quarks. However, this $T$ is small: not only is it associated with weakboson exchange and thus naturally suppressed at low energies ( $\propto G_{F}$ at least), but it also appears in a small combination of CKM elements, $J_{C P} \simeq 3 \cdot 10^{-5}$.

Because $T$ is not a symmetry of the Standard Model, other $T$ operators must exist, which are allowed by all the symmetries of the theory. The best known of such interactions is the only

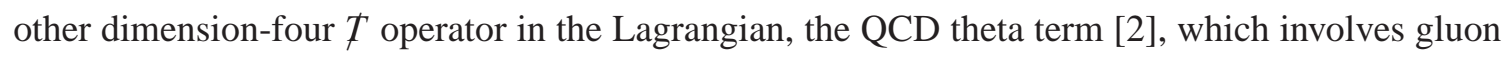
fields and is parametrized by the vacuum angle $\bar{\theta}$. Despite being a total derivative, it contributes to observables due to topological effects.

The effects of such $T$ sources have long been sought in flavor-conserving processes, in particular permanent electric dipole moments (EDMs) of light elementary particles. Because then the EDM has to be proportional to the spin, $\vec{d}=d \vec{S} /|\vec{S}|$, the interaction with an electric field $\vec{E}, \vec{d} \cdot \vec{E}$, changes sign under both $P$ and $T$. For particles of spin one or larger, further $T$ moments exist; relevant for light nuclei, which can have spin one, is the magnetic quadrupole moment (MQM), which is also parity violating $(\boldsymbol{P})$. Associated with an electromagnetic moment there is a form factor - for example the EDFF and the MQFF - which incorporates the momentum dependence of the interaction between the particle and the photon. The EDFF, in particular, is of interest both theoretically and experimentally. For a virtual photon, the term linear in the square of the photon momentum cannot be distinguished from a short-range interaction, called the Schiff moment (SM) $S^{\prime}$; a nuclear SM contributes, for example, to atomic EDMs. Moreover, in some lattice QCD calculations, because of infrared regularization, it is the EDFF at finite momentum, not directly the EDM, that is calculated.

Many years of attempts to directly measure the EDM of the neutron have led to the stringent bound $d_{n}=0.2 \pm 1.5 \pm 0.7 \cdot 10^{-13} e \mathrm{fm}$ [阿. Efforts are underway in several laboratories with the aim to increase the sensitivity to $10^{-15} e \mathrm{fm}$, for example at SNS and ILL [4]. For the proton, the bound is indirect, $\left|d_{p}\right|<7.9 \cdot 10^{-12} e$ fm $[5]$, coming from the absence of atomic EDMs and using input from nuclear-structure calculations [6].

The new, exciting possibility is the direct measurement of the EDMs of charged particles in storage rings, possibly at BNL and COSY [7]. When a particle moves in an electric and/or magnetic 
field, its spin will precess at a rate that depends not only on the magnetic dipole moment but also on the EDM. The best bound on the muon EDM comes, in fact, as a by-product on the BNL $g-2$ experiment [8]. It can be expected that dedicated experiments in rings with optimized parameters (in particular for $\vec{E}$ ) will allow sensitive probing of the EDMs of light nuclei. For example, it has been proposed [7] that for $d_{p}$ a sensitivity of $10^{-16} e$ fm can be achieved. Similar sensitivity could be attained also for the deuteron and helion ( ${ }^{3} \mathrm{He}$ nucleus) EDMs, $d_{d}$ and $d_{h}$ respectively. I will argue below that the practically more complicated case of the triton ( ${ }^{3} \mathrm{H}$ nucleus) EDM, $d_{t}$, should also be considered. And, while it is likely not doable in the near future at the required level, access to the deuteron MQM $\left(\mathscr{M}_{d}\right)$ would be very useful as well.

This considerable experimental progress opens a wide window to new physics. The contribution of the CKM phase to such flavor-conserving observables is estimated to be very small, for example $d_{n} \simeq 10^{-19} e \mathrm{fm}$ [1]. Thus, any signal observed in the new generation of experiments must come from other sources of $T$. The stringent bound on $d_{n}$ already limits the QCD vacuum angle to a very unnaturally small value $\left(\bar{\theta} \lesssim 3 \cdot 10^{-11}\right)$, the origin of which is not understood. As a consequence, $T$ sources of higher canonical dimension might be more important than the dimension-four sources. Lacking other information, we expect these to be the operators of lowest dimension, that is, six [9]: operators associated with the quark EDM (qEDM), quark and gluon color EDMs (qCEDM and gCEDM), and 7 four-quark interactions (4QOs).

The issue I want to discuss here is: once a hadronic or nuclear EDM is observed, how many and which observables do we need to identify the dominant $T$ source(s)? The strategy I will follow is to consider effective field theories (EFTs) at successively lower energy scales (Sect. 2), then use the one applicable at the nuclear scale to calculate the $P, T$ electromagnetic moments of light nuclei (Sect. B). Although many holes still need to be filled and extensions to be launched, one can conclude (Sect. \#) that the proposed experimental program on light nuclear EDMs has the potential to identify the most important $T$ sources of higher dimension.

\section{Interactions}

The Standard Model is an EFT for processes at momenta $Q \lesssim M_{Z}$, the mass of the $Z$ boson standing here for the characteristic electroweak scale. This EFT comprises all the degrees of freedom with masses of the same order or smaller, with interactions constrained by an $S U(3)_{c} \times$ $S U(2)_{L} \times U(1)_{Y}$ gauge symmetry. One expects that the higher the canonical dimension of an operator, the more it is suppressed by the relatively high scale $M_{\mathbb{T}^{\prime}}$ where unknown physics become important and the electroweak EFT breaks down. At $Q \sim M_{Z}$, the unknown physics is captured predominantly by dimension-six operators involving Standard Model fields and symmetries. As we lower the typical momentum of interest, $Q \lesssim M_{Z}$, we can run the renormalization group, integrating out the heaviest Standard Model particles and using perturbation theory to compute the change in the coupling strengths with the scale, until at a scale $M_{Q C D} \sim 1 \mathrm{GeV}$ strong interactions become non-perturbative.

Here I focus on strongly interacting particles and denote the two-flavor quark field by $q=$ $(u d)^{T}$, the gluon and photon field strengths by $G_{\mu \nu}$ and $F_{\mu \nu}$, and their duals by $\tilde{G}_{\mu \nu}$ and $\tilde{F}_{\mu \nu}$, respectively. The three Pauli matrices in isospin space are written $\tau$ and the eight Gell-Mann 
matrices in color space, $\underline{\lambda}$. Then the $T$ effective Lagrangian just above $M_{Q C D}$ can be written as

$$
\begin{aligned}
\mathscr{L}_{Q C D, \not J}= & \frac{\bar{m}}{2}\left(1-\varepsilon^{2}\right) \bar{\theta} \bar{q} i \gamma_{5} q-\frac{1}{2} \bar{q}\left(c_{q}^{(0)}+c_{q}^{(1)} \tau_{3}\right) \sigma_{\mu v} \tilde{G}^{\mu v} q-\frac{1}{2} \bar{q}\left(d_{q}^{(0)}+d_{q}^{(1)} \tau_{3}\right) \sigma_{\mu v} \tilde{F}^{\mu v} q \\
& +c_{G} \operatorname{Tr}\left(G_{\mu}{ }^{\rho} G_{\rho v} \tilde{G}^{\mu v}\right)+C_{1}\left(\bar{q} q \bar{q} i \gamma_{5} q-\bar{q} \tau q \cdot \bar{q} i \gamma_{5} \tau q\right) \\
& +C_{8}\left(\bar{q} \underline{\lambda} q \cdot \bar{q} i \gamma_{5} \underline{\lambda} q-\bar{q} \tau \underline{\lambda} q \cdot \cdot \bar{q} i \gamma_{5} \tau \underline{\lambda} q\right)+\ldots
\end{aligned}
$$

Here the first term is the theta term, already incorporating the smallness of $\bar{\theta}$ and a chiral rotation [10] that eliminates the purely gluonic term via the axial anomaly and enforces vacuum stability to first order in the quark masses. A chiral rotation affects the quark mass terms, responsible for an average quark mass $\bar{m}=\left(m_{u}+m_{d}\right) / 2$ and a quark mass difference $m_{d}-m_{u}=2 \varepsilon \bar{m}$. The second and third terms represent, respectively, isoscalar $\left(c_{q}^{(0)}\right)$ and isovector $\left(c_{q}^{(1)}\right)$ qCEDMs, and isoscalar $\left(d_{q}^{(0)}\right)$ and isovector $\left(d_{q}^{(1)}\right)$ qEDMs. They originate from dimension-six operators at $Q \sim M_{Z}$, as in order to couple gluon and electroweak bosons to quarks and satisfy the gauge symmetries, one needs also a Higgs field [0]. Trading the Higgs vacuum expectation value for $\bar{m}$ and the average Yukawa coupling $f$ of the Higgs to the light quarks, and denoting the proton charge by $e=\sqrt{4 \pi \alpha}$ and the original interaction strengths by $4 \pi g$ for qCEDM and $4 \pi \breve{g}$ for qEDM,

$$
c_{q}^{(i)}=\mathscr{O}\left(\frac{4 \pi g \bar{m}}{f M_{T^{\prime}}^{2}}\right), \quad d_{q}^{(i)}=\mathscr{O}\left(\frac{e \breve{g} \bar{m}}{f M_{T^{\prime}}^{2}}\right) .
$$

The fourth, fifth, and sixth terms are the dimension-six gCEDM and two 4QOs allowed by the Standard Model structure [9]. Writing their coefficients as

$$
c_{G}=\mathscr{O}\left(\frac{4 \pi w}{M_{T^{\prime}}^{2}}\right), \quad C_{i}=\mathscr{O}\left(\frac{(4 \pi)^{2} \sigma_{i}}{M_{T^{\prime}}^{2}}\right),
$$

the dimensionless parameters $w$ and $\sigma_{i}$ are expected by naive dimensional analysis (NDA) to be $\mathscr{O}(1)$. Finally, the "..." stand for terms not explicitly considered in my talk. They include one other dimension-six contribution, recently pointed out in Ref. [11], the rest being higher-order operators suppressed by higher powers of $M_{\mathbb{T}^{\prime}}^{-1}$.

Note that up to dimension six, $T$ implies $\not P$, and all these operators can contribute linearly to EDMs. But how? In order to calculate their contributions to hadronic quantities we need to solve QCD at low energies, a notoriously difficult problem. Once this is done, the results can be matched onto those of an EFT designed for $Q<M_{Q C D}$, called chiral perturbation theory $(\chi \mathrm{PT})$, which involves the lightest hadrons - the nucleon isodoublet $(N)$ and the pion isotriplet $(\boldsymbol{\pi})$ - and the photon. This EFT implements the fact that the approximate chiral symmetry of QCD, $S U(2)_{L} \times$ $S U(2)_{R} \sim S O(4)$, must be spontaneously broken to its diagonal, isospin subgroup, $S U(2)_{L+R} \sim$ $S O(3)$. The pions are pseudo-Goldstone bosons with a decay constant $f_{\pi} \sim M_{Q C D} / 4 \pi$ and a small mass

$$
m_{\pi}^{2}=\mathscr{O}\left(\bar{m} M_{Q C D}\right)
$$

Nucleons realize chiral symmetry non-linearly and have a mass $m_{N} \sim M_{Q C D}$. Lorentz invariance is most easily incorporated in a $Q / m_{N}$ expansion by employing a heavy nucleon field, which is a Pauli spinor of spin $S_{\mu}$ and velocity $v_{\mu}$. No nucleon-pair creation needs to be included explicitly, as 
these effects are integrated out together with other physics of momenta above $M_{Q C D}$. Observables are expressed in powers of $Q / M_{Q C D}$ and parametrized by low-energy constants (LECs) that can in principle be determined from lattice QCD.

The crucial idea that I want to explore is that each 7 term in Eq. (2.1) transforms under chiral symmetry in a particular way. Thus, while each produces interactions among nucleons, pions and photons that break $T$, the specific form and magnitude of these interactions depends on the $T$ source. Given enough low-energy observables it should be possible to disentangle the various sources.

The $T$ interactions in two-flavor $\chi \mathrm{PT}$ have been constructed in detail [12]. The theta term transforms as the fourth component of the same chiral four-vector whose third component is responsible for quark-mass isospin violation $(\not)$. There is thus a generic relation between $T$ from $\bar{\theta}$ and $T$-conserving $\not$. The qCEDM and qEDM each transform as third and fourth components of two chiral vectors. The gCEDM and the two 4QOs are chiral invariant operators ( $\chi$ IOs) and thus cannot be separated at low energies, and I will address them together with the shorthand notation

$$
\left\{w, \sigma_{1}, \sigma_{8}\right\} \rightarrow w .
$$

It turns out that as far as light nuclear EDMs are concerned, in leading order (LO) only six $T$ interactions are relevant,

$$
\begin{aligned}
\mathscr{L}_{\chi P T, \mathbb{T}}= & -2 \bar{N}\left(\bar{d}_{0}+\bar{d}_{1} \tau_{3}\right) S_{\mu} N v_{v} F^{\mu v}-\frac{1}{2 f_{\pi}} \bar{N}\left(\bar{g}_{0} \boldsymbol{\tau} \cdot \boldsymbol{\pi}+\bar{g}_{1} \pi_{3}\right) N \\
& +\bar{C}_{1} \bar{N} N \partial_{\mu}\left(\bar{N} S^{\mu} N\right)+\bar{C}_{2} \bar{N} \tau N \cdot \partial_{\mu}\left(\bar{N} S^{\mu} \tau N\right)+\ldots
\end{aligned}
$$

The first term represents isoscalar $\left(\bar{d}_{0}\right)$ and isovector $\left(\bar{d}_{1}\right)$ short-range contributions to the nucleon EDM. The second term is the $\not{P}, T$ pion-nucleon coupling, consisting likewise of isoscalar $\left(\bar{g}_{0}\right)$ and isovector $\left(\bar{g}_{1}\right)$ pieces. The third and fourth terms are $\not P, T$ two-nucleon contact interactions. The "..." subsume an infinite number of other $P, T$ interactions: terms related to the above by chiral symmetry, and interactions involving larger number of derivatives and nucleon fields, and more powers of small parameters —all of which only contribute to light nuclear EDMs at next-toleading order (NLO) or higher, but one does contribute to the MQM at LO.

Where are the differences among $T$ sources? First, they are in the different forms hidden in the "..." of Eq. (2.6), and will show up in subleading orders. Second, for a given source not all interactions shown explicitly above appear in LO. As an example, consider the pion-nucleon coupling, where by NDA

$$
\begin{aligned}
& \bar{g}_{0}=\mathscr{O}\left(\bar{\theta} \frac{m_{\pi}^{2}}{M_{Q C D}}, \frac{g}{f} \frac{m_{\pi}^{2} M_{Q C D}}{M_{\mathbb{T}^{\prime}}^{2}}, \frac{\breve{g}}{f} \frac{\alpha}{\pi} \frac{m_{\pi}^{2} M_{Q C D}}{M_{\mathbb{T}^{\prime}}^{2}}, w \frac{m_{\pi}^{2} M_{Q C D}}{M_{\mathbb{T}^{\prime}}^{2}}\right), \\
& \bar{g}_{1}=\mathscr{O}\left(\bar{\theta} \frac{m_{\pi}^{4}}{M_{Q C D}^{3}}, \frac{g}{f} \frac{m_{\pi}^{2} M_{Q C D}}{M_{\mathbb{T}^{\prime}}^{2}}, \frac{\breve{g}}{f} \frac{\alpha}{\pi} \frac{m_{\pi}^{2} M_{Q C D}}{M_{\mathbb{T}^{\prime}}^{2}}, w \frac{m_{\pi}^{2} M_{Q C D}}{M_{\mathbb{T}^{2}}^{2}}\right) .
\end{aligned}
$$

For $\bar{\theta}, \bar{g}_{1}$ is as subleading as two-derivative pion-nucleon interactions. The link with $\not /$ means the strong-interaction matrix element in $\bar{g}_{0}$ is related to the nucleon mass difference $\left(m_{n}-m_{p}\right)_{q m}$ stemming from quark mass splitting: $\bar{g}_{0} \simeq\left(m_{n}-m_{p}\right)_{q m} \bar{\theta} / 2 \varepsilon \approx 3 \bar{\theta} \mathrm{MeV}$ [12]. For the dimensionsix sources both structures appear at the same order, but for qEDM the $\alpha / \pi$ suppression renders 
pion physics higher order, and for $\chi \mathrm{IOs}$ the $m_{\pi}^{2}$ factor implies they appear at the same level as chiralinvariant two-derivative pion-nucleon interactions. Note that for all these sources a third structure, the isotensor $\bar{N} \pi_{3} \tau_{3} N$ [ [13], is of higher order. Frequently $T$ calculations in nuclear physics are parametrized solely in terms of these three pion-nucleon coupling structures, while EFT suggests that for EDMs one should use the six LECs from Eq. (2.6) instead.

\section{Electric Dipole Moments}

EDMs of the nucleon and light nuclei can now be calculated using $\chi$ PT. Here I show only orders of magnitude; for detailed formulas that give the dependence on the various LECs, please consult the references cited below.

The full nucleon EDFF has been calculated at LO [14] and NLO [15] for all sources described above. Results are sketched in Table 11. The short-range EDMs $\bar{d}_{i}$ appear at LO for all sources, but are not yet known from lattice QCD (for $\bar{\theta}$, see Ref. [16]). The order where a one-pion loop brings in a long-range contribution from $\bar{g}_{i}$ (or a higher-order correction) depends on the source. For $\bar{\theta}$ and qCEDM, it is LO and for the former, assuming no cancellations against short-range physics, one can estimate $\left|d_{N}\right| \gtrsim 2 \cdot 10^{-3} \bar{\theta} e$ fm [14], from which the current bound on $\bar{\theta}$ arises. For the other sources, from NDA, we obtain $g / f M_{\mathbb{T}^{\prime}}^{2}, \breve{g} / f M_{T^{\prime}}^{2} \lesssim\left(10^{5} \mathrm{GeV}\right)^{-2}$ and $w / M_{\mathbb{T}^{\prime}}^{2} \lesssim\left(10^{6} \mathrm{GeV}\right)^{-2}$, showing that we probe LHC-caliber scales. However, the LO existence of at least two a priori unknown LECs means that $\left|d_{n}\right|$ and $\left|d_{p}\right|$ can be expected to be of similar size, and their measurement could be fitted by any one $T$ source. The SMs could in principle allow a partial separation of sources, but there is no obvious way to determine them.

We are thus led to consider light nuclei, where a resummation of certain $T$-conserving interactions is needed to produce bound states and their associated wavefunctions. A nuclear EDM consists of the average with such a wavefunction of the $T$ current [17, 18, 19, and of a combination of $T$ potential [20] and $T$-conserving current. LO results are sketched in Table 2 [17, 18, 19] for the deuteron of mass $m_{d}=m_{p}+m_{n}-B_{d}$, and in Table 3 [18] for helion and triton of masses $m_{h}$ and $m_{t}$, respectively.

For $\bar{\theta}$, assuming no fine-tuning, $\left|d_{d}\right| \gtrsim 3 \cdot 10^{-4} \bar{\theta} e$ fm from the long-range contributions to the isoscalar nucleon EDM, which appear at NLO [15]. A bound on $\left|d_{d}\right|$ at the proposed level would strengthen the bounds on $T$ parameters to $\bar{\theta} \lesssim 3 \cdot 10^{-13}, g / f M_{T}^{2}, \breve{g} / f M_{\mathbb{T}}^{2} \lesssim\left(5 \cdot 10^{6} \mathrm{GeV}\right)^{-2}$ and $w / M_{T^{\prime}}^{2} \lesssim\left(3 \cdot 10^{7} \mathrm{GeV}\right)^{-2}$. Since $d_{d} \simeq d_{p}+d_{n}$ for $\bar{\theta}, \mathrm{qEDM}$ and $\chi \mathrm{IOs}$, a measurement of $d_{d}$ alone could again be fitted with any one source, unless $\left|d_{d}\right|$ is large compared to $\left|d_{N}\right|$, which would be

\begin{tabular}{c|cccc}
\hline \hline Source & $\bar{\theta}$ & qCEDM & qEDM & $\chi$ IOs \\
\hline$m_{N} d_{n} / e$ & $\bar{\theta} m_{\pi}^{2} / M_{Q C D}^{2}$ & $(g / f)\left(m_{\pi}^{2} / M_{T^{\prime}}^{2}\right)$ & $(\breve{g} / f)\left(m_{\pi}^{2} / M_{\mathbb{T}}^{2}\right)$ & $w M_{Q C D}^{2} / M_{\mathbb{T}}^{2}$ \\
$d_{p} / d_{n}$ & 1 & 1 & 1 & 1 \\
$4 m_{\pi}^{2} S_{p}^{\prime} / d_{p}$ & 1 & 1 & $m_{\pi}^{2} / M_{Q C D}^{2}$ & $m_{\pi}^{2} / M_{Q C D}^{2}$ \\
$4 m_{\pi}^{2} S_{N}^{\prime(0)} / d_{n}$ & $m_{\pi} / M_{Q C D}$ & $m_{\pi} / M_{Q C D}$ & $m_{\pi}^{2} / M_{Q C D}^{2}$ & $m_{\pi}^{2} / M_{Q C D}^{2}$ \\
\hline \hline
\end{tabular}

Table 1: Orders of magnitude for the neutron EDM (in units of $e m_{N}^{-1}$ ), the proton-to-neutron EDM ratio, the proton SM-to-EDM ratio (in units of $\left(2 m_{\pi}\right)^{-2}$ ), and the ratio of isoscalar SM to neutron EDM (in units of $\left(2 m_{\pi}\right)^{-2}$ ), for $T$ sources of effective dimension up to six. 


\begin{tabular}{c|cccc}
\hline \hline Source & $\bar{\theta}$ & qCEDM & qEDM & $\chi$ IOs \\
\hline$m_{d} d_{d} / e$ & $\bar{\theta} m_{\pi}^{2} / M_{Q C D}^{2}$ & $(g / f)\left(M_{Q C D}^{2} / M_{T^{\prime}}^{2}\right)$ & $(\breve{g} / f)\left(m_{\pi}^{2} / M_{T^{\prime}}^{2}\right)$ & $w M_{Q C D}^{2} / M_{\mathbb{T}}^{2}$ \\
$d_{d} / d_{n}$ & 1 & $M_{Q C D}^{2} / m_{\pi}^{2}$ & 1 & 1 \\
$8 m_{d} B_{d} S_{d}^{\prime} / d_{d}$ & 1 & 1 & 1 & 1 \\
$m_{d} \mathscr{M}_{d} / d_{d}$ & $M_{Q C D}^{2} / m_{\pi}^{2}$ & 1 & $\sqrt{m_{d} B_{d} / 2 m_{\pi}^{2}}$ & 1 \\
\hline \hline
\end{tabular}

Table 2: Orders of magnitude for the deuteron EDM (in units of $e m_{d}^{-1}$ ), the deuteron-to-neutron EDM ratio, the deuteron SM-to-EDM ratio (in units of $\left.\left(8 m_{d} B_{d}\right)^{-1}\right)$ and the deuteron MQM-to-EDM ratio (in units of $\left.m_{d}^{-1}\right)$, for $T$ sources of effective dimension up to six.

suggestive of qCEDM. The SM adds no new information, but the MQM, if it could be measured, might isolate $\bar{\theta}$ or qEDM. For the former, $\left|\mathscr{M}_{d}\right| \simeq 2 \cdot 10^{-3} \bar{\theta} e \mathrm{fm}^{2}$ at LO, entirely from long-range contributions.

Lacking a potential measurement of $\mathscr{M}_{d}$, we go farther on to helion and triton. A large trinucleon EDM compared to a nucleon EDM would point to $\bar{\theta}$ and qCEDM as dominant sources. Thus, $d_{n}, d_{d}$, and $d_{h}$ could identify whether $\bar{\theta}$ or qCEDM is most important. And, since $d_{h}+d_{t} \simeq$ $3 d_{d}$ for qCEDM, $d_{h}+d_{t} \simeq 0.84\left(d_{n}+d_{p}\right)$ for $\bar{\theta}$ and qEDM, and $d_{h}-d_{t} \simeq 0.94\left(d_{n}-d_{p}\right)$ for qEDM, adding knowledge of $d_{p}$ and $d_{t}$ could isolate qEDM as well. The chiral structure of the various sources can therefore be separated in the proposed experimental program.

\section{Conclusion and Outlook}

I have argued that a QCD-based framework exists for the calculation of nuclear $T$ observables. Chiral symmetry properties determine the form and size of the low-energy $T$ interactions. As a consequence, the pattern of nucleon, deuteron, helion, and triton $T$ form factors partially reflects the underlying $T$ source. The experimental program to measure light nuclear EDMs could teach us significant lessons about physics beyond the Standard Model.

However, much still remains to be done, even at the theoretical level, to strengthen these arguments. First, nuclear EDMs at NLO are necessary to test convergence of the EFT, which has already been shown to converge well for the nucleon [15]. Second, the LECs should be calculated in lattice QCD for each source, to lessen the reliance on NDA. Third, the runnings of all sources from $M_{Z}$ to $M_{Q C D}$ is needed for more quantitative statements. Finally, in another direction, one wonders if the EDFFs of larger nuclei could be calculated in terms of the same six LECs needed in light nuclei. There is plenty to keep us awake in the city of lights.

\begin{tabular}{c|cccc}
\hline \hline Source & $\bar{\theta}$ & qCEDM & qEDM & $\chi$ IOs \\
\hline$m_{h} d_{h} / e$ & $\bar{\theta}$ & $(g / f)\left(M_{Q C D}^{2} / M_{T^{\prime}}^{2}\right)$ & $(\breve{g} / f)\left(m_{\pi}^{2} / M_{T^{2}}^{2}\right)$ & $w M_{Q C D}^{2} / M_{T^{2}}^{2}$ \\
$d_{t} / d_{h}$ & 1 & 1 & 1 & 1 \\
$d_{h} / d_{n}$ & $M_{Q C D}^{2} / m_{\pi}^{2}$ & $M_{Q C D}^{2} / m_{\pi}^{2}$ & 1 & 1 \\
\hline \hline
\end{tabular}

Table 3: Orders of magnitude for the helion EDM (in units of $e m_{h}^{-1}$ ), the triton-to-helion EDM ratio, and the helion-to-neutron EDM ratio, for $T$ sources of effective dimension up to six. 


\section{Acknowledgments}

I thank Jordy de Vries, Emanuele Mereghetti, Renato Higa, Will Hockings, CP Liu, Claudio Maekawa, Ionel Stetcu, and Rob Timmermans for enjoyable collaborations on the work described here, and the first two for comments on the manuscript. I am grateful to KVI/Groningen for hospitality. This research was supported in part by the US DOE under grant DE-FG02-04ER41338.

\section{References}

[1] M. Pospelov and A. Ritz, Ann. Phys. 318 (2005) 119.

[2] G. 't Hooft, Phys. Rev. Lett. 37 (1976) 8.

[3] C.A. Baker et al., Phys. Rev. Lett. 97 (2006) 131801.

[4] J.-C. Peng, Mod. Phys. Lett. A 23 (2008) 1397.

[5] W.C. Griffith et al., Phys. Rev. Lett. 102 (2009) 101601.

[6] V.F. Dmitriev and R.A. Sen'kov, Phys. Rev. C 71 (2005) 035501.

[7] Y.K. Semertzidis, arXiv:1110.3378; C.J.G. Onderwater, arXiv:1204.2512.

[8] G.W. Benett et al., Phys. Rev. D 80 (2009) 052008.

[9] W. Buchmüller and D. Wyler, Nucl. Phys. B 268 (1986) 621; S. Weinberg, Phys. Rev. Lett. 63 (1989) 2333; A. De Rújula, M.B. Gavela, O. Pène, and F.J. Vegas, Nucl. Phys. B 357 (1991) 311; M.J.

Ramsey-Musolf and S. Su, Phys. Rept. 456 (2008) 1; B. Grzadkowski, M. Iskrzynski, M. Misiak, and J. Rosiek, JHEP 1010 (2010) 085.

[10] V. Baluni, Phys. Rev. D 19 (1979) 2227.

[11] J. Ng and S. Tulin, Phys. Rev. D 85 (2012) 033001.

[12] E. Mereghetti, W.H. Hockings, and U. van Kolck, Ann. Phys. 325 (2010) 2363; J. de Vries, E. Mereghetti, R. Timmermans, and U. van Kolck, in preparation.

[13] G. Barton, Nuovo Cim. 19 (1961) 512.

[14] R.J. Crewther, P. Di Vecchia, G. Veneziano, and E. Witten, Phys. Lett. B 88, 123 (1979); 91, 487(E) (1980); S.D. Thomas, Phys. Rev. D 51 (1995) 3955; W.H. Hockings and U. van Kolck, Phys. Lett. B 605 (2005) 273; J. de Vries, R.G.E. Timmermans, E. Mereghetti, and U. van Kolck, Phys. Lett. B 695 (2011) 268.

[15] S. Narison, Phys. Lett. B 666 (2008) 455; K. Ottnad, B. Kubis, U.-G. Meißner, and F.-K. Guo, Phys. Lett. B 687 (2010) 42; E. Mereghetti, J. de Vries, W.H. Hockings, C.M. Maekawa, and U. van Kolck, Phys. Lett. B 696 (2011) 97.

[16] F. Berruto, T. Blum, K. Orginos, and A. Soni, Phys. Rev. D 73 (2006) 054509; E. Shintani, S. Aoki, and Y. Kuramashi, Phys. Rev. D 78 (2008) 014503.

[17] I.B. Khriplovich and R.A. Korkin, Nucl. Phys. A 665 (2000) 365; J. de Vries, E. Mereghetti, R.G.E. Timmermans, and U. van Kolck, Phys. Rev. Lett. 107 (2011) 091804.

[18] J. de Vries, R. Higa, C.-P. Liu, E. Mereghetti, I. Stetcu, R.G.E. Timmermans, and U. van Kolck, Phys. Rev. C 84 (2011) 065501.

[19] C.-P. Liu, J. de Vries, E. Mereghetti, R.G.E. Timmermans, and U. van Kolck, Phys. Lett. B (to appear), arXiv:1203.1157 [hep-ph].

[20] C.M. Maekawa, E. Mereghetti, J. de Vries, and U. van Kolck, Nucl. Phys. A 872 (2011) 117. 